\title{
Enhancing the usability of usability design tools: The case of information requirements specification
}

\author{
Juliet Richardson and Thomas C. Ormerod \\ Department of Psychology, Lancaster University, Lancaster, LA1 4YF, U.K. \\ RichardsonJ@Cardiff.ac.uk, T.Ormerod@Lancaster.ac.uk
}

\begin{abstract}
In this paper we report the development of a tool for information requirements specification (IRS), designed to support the application of a particular IRS methodology, the Sub-Goal Template scheme (Shepherd, 1993; Shepherd and Ormerod, 1992), and the use of a notation embodied within that methodology. First, an empirical study was conducted to determine typical errors made by users of the IRS notation which was subsequently re-designed to avoid these errors through the provision of templates. Second, the tasks involved in using the IRS methodology were articulated in order to develop a process model for a computer-based support tool. Finally, an evaluation of the usability of the tool showed that it achieved the main aims of supporting both the notation and the methodology. We argue that, in developing methods that address usability issues, the usability of design aids to support the methodologies should also be subject to scrutiny.
\end{abstract}

KEY WORDS Usability, Methodologies, Notations, Requirements Specification, Design Support Tools

\section{INTRODUCTION}

A large number of tools and methodologies have been developed to support complex activities such as design. However, the notations and representations embodied in such supporting technologies are rarely subjected to close scrutiny. In this paper, we report a number of studies that examine the usability of a notation for information requirements specification (IRS). The IRS methodology is based upon the SubGoal Template scheme of Shepherd (1993; see also Shepherd and Ormerod, 1992), which employs a variant of Hierarchical Task Analysis (HTA: Annett, Duncan, Stammers and Gray, 1971), to identify stereotypical user tasks and their associated information needs at a level of detail that is useful but not overly constraining for subsequent interface design. The studies resulted in the development of a computer-based IRS tool which delivers information requirements in a form that can be used as a tendering document for interface design.

Design in domains such as architecture, engineering and software development is generally conceptualised as a complex problem-solving activity (Simon, 1969), entailing processes such as problem definition and decomposition, and solution development and simulation. The extremely ill-defined nature of design tasks, and the time lag between the specification of requirements and feedback upon the viability of solutions, have been proposed to differentiate design from other kinds of problem-solving activity (Goel and Pirolli, 1989). In order to support designers, many tools and methodologies have been developed (e.g. Ashworth and Goodland, 1990; Jackson, 1980). Typically such supporting technologies are developed by domain specialists, and tend to reflect a focus upon desirable characteristics of a design product rather than an understanding of the design process (Guindon, 1992; Lansdale and Ormerod, 1994). Whilst psychologists have increasingly been involved in the development of design methodologies, to date there has been little psychological research into the utility of the notations underlying such methodologies. 
Our research into design support technologies has focused upon the development of an IRS methodology for process control displays, that is, identifying the information that an operator needs to undertake each task entailed in the control of plant and equipment. Identifying information requirements is an inherently psychological task and one that is critical for effective display design, yet it is rarely given the prominence it deserves. This is in part because designers tend to focus upon specifying functional requirements for displays, and also because it entails expertise in human factors.

In the domain of interface design there are four essential characteristics of a requirements specification:

(1) The requirements specification should be complete in order that the final system designed will support the whole range of operator tasks.

(2) Both the functional requirements of the system and the information requirements of the human operator(s) should be specified. However, information requirements are often neglected and many methodologies focus only on functional requirements (Harker, Olphert and Eason, 1990). Interface design can end up being an afterthought which is considered only after the rest of the system has been designed.

(3) The requirements specification should be independent of details arising from any existing or potential implementations (Parnas and Clements, 1986). This will allow the designers to freely choose the best solutions for the design.

(4) The process of requirements specification needs some degree of guidance and formalism. This can be achieved through following formal design methodologies. Such methodologies provide a structure within which the design process can occur, helping break the design task down into manageable units.

There are numerous methodologies which aim to support the process of requirements specification (see Appendix A of Olle, Hagelstein, Macdonald, Rolland, Sol, Van Assche and Verrijn-Stuart, 1988 for an overview of 34 methodologies). However in this paper we shall focus on specific issues by considering only one methodology, the Sub-Goal Template (SGT) scheme as developed by Shepherd and Ormerod (1992; Shepherd, 1993) to support information requirements specification for process control interfaces.

\section{THE SGT SCHEME}

The SGT scheme sets out to meet the needs outlined above by taking an HTA as the basis for a requirements specification. HTA, developed by Annett et al. (1971), is a well established technique for task analysis. An HTA involves identifying the immediate subgoals of a task. Each of these subgoals can then be taken in turn and themselves redescribed in terms of subgoals. This redescription process is repeated until the level of the analysis is of a fine enough grain to be useful for design. However, care must be taken that the task analysis is not a description of the existing technology: the analysis should cease at a point where the tasks describe simple implementation-independent operations such as "Open valve C" rather than specific tasks such as "Press button B" which are tied in to the existing interface. Not only does an HTA consist of a detailed redescription of tasks but it also contains plans that describe relationships between the tasks and constraints acting on them. Plans allow sequence information to be captured which can then be used to determine the organisation of information requirements.

The SGT scheme provides a notation for taking the output from an HTA and using it as the basis for an IRS. There are two parts to this process.

(1) Firstly, the information requirements of the lowest level tasks in the HTA are specified through the use of templates known as SGT elements. Each task is redescribed using the SGT notation as an instance of a more generalised stereotypical task, such as "Adjust equipment" or "Read" or "Monitor rate of change". For example, the task of altering the opening of a valve might be classified as "Adjust equipment" as might the task of altering the temperature of a liquid or of varying a pipe flowrate. Each of these tasks has associated stereotypical information needs. For example, in order to carry out the task "Adjust equipment" the operator would need to know the possible operating states of the equipment together with feedback confirming its actual state. In this way the information requirements for each operational task can be specified.

(2) In addition to the information requirements the SGT scheme also provides a notation for specifying the organisation of that information. This is achieved by taking the plans from the HTA, which detail sequences and interdependencies between tasks, and re-writing them using the sequencing elements of the SGT scheme. There are four of these elements as shown in Table 1. The designer can use the information captured by these sequencing elements to ensure that the organisation of information on the interface is optimal. For example, information required for tasks that are performed at the same time should be displayed in one location. 


\begin{tabular}{|ll|}
\hline \multicolumn{2}{|c|}{ Sequencing elements. } \\
\hline Code & Label \\
S1 & fixed procedure: then do \\
S2 & choice: if $Z$ then do $X$ if not $Z$ then do $Y$ \\
S3 & parallel: then do together $X$ and $Y$ \\
S4 & optional sequence: do in any order $X$ and $Y$ \\
\hline
\end{tabular}

Table 1. The sequencing elements (from Shepherd and Ormerod, 1992). Note: $X$ and $Y$ refer to tasks and $Z$ refers to an operation condition.

Thus the SGT scheme provides a notation which can be used to redescribe an HTA to produce an information requirements specification. It not only specifies the full set of information required by the operator but also gives some detail of how to organise that information. The SGT scheme meets three of the four pre-requisites for a successful requirements specification given above: firstly, the specification should be complete as long as it is based on a complete HTA; the specification should be independent from implementation as it is based on an HTA that focuses on tasks and goals rather than on the specific mechanisms used to achieve those goals; the specification of information requirements is at the heart of the SGT scheme and is therefore not sidelined. However, by itself the SGT notation does not really give any guidance to users or introduce any formalism into the process of design. This can only be achieved by a methodology.

A methodology for using the SGT scheme is shown in Figure 1. There are two ways in which the SGT scheme can be used to produce an information requirements specification: an existing HTA can be translated into SGT elements, or an HTA can be constructed from scratch using SGT elements. Either process will produce a formal analysis of the operators' tasks together with the information needs of these tasks.

The first stage in producing an SGT-based IRS is to select a goal. This will either be the initial goal in a plan or the next goal in the plan currently being explored. Once a goal has been selected the analyst must first decide whether that goal should be assigned to the system or to the operator. If the goal can be automated then it is assigned to the system; if not then it is assigned to the operator and the analyst must then attempt to redescribe the goal as a single SGT element. If this is possible then the appropriate SGT is recorded, the information requirements are added and the next goal, if any, is identified.

If redescription as a single SGT element is not possible then the current goal must be decomposed into a set of subgoals governed by a plan. The plan should be hypothesised in terms of sequencing elements and evaluated against the goal constraints. Once an

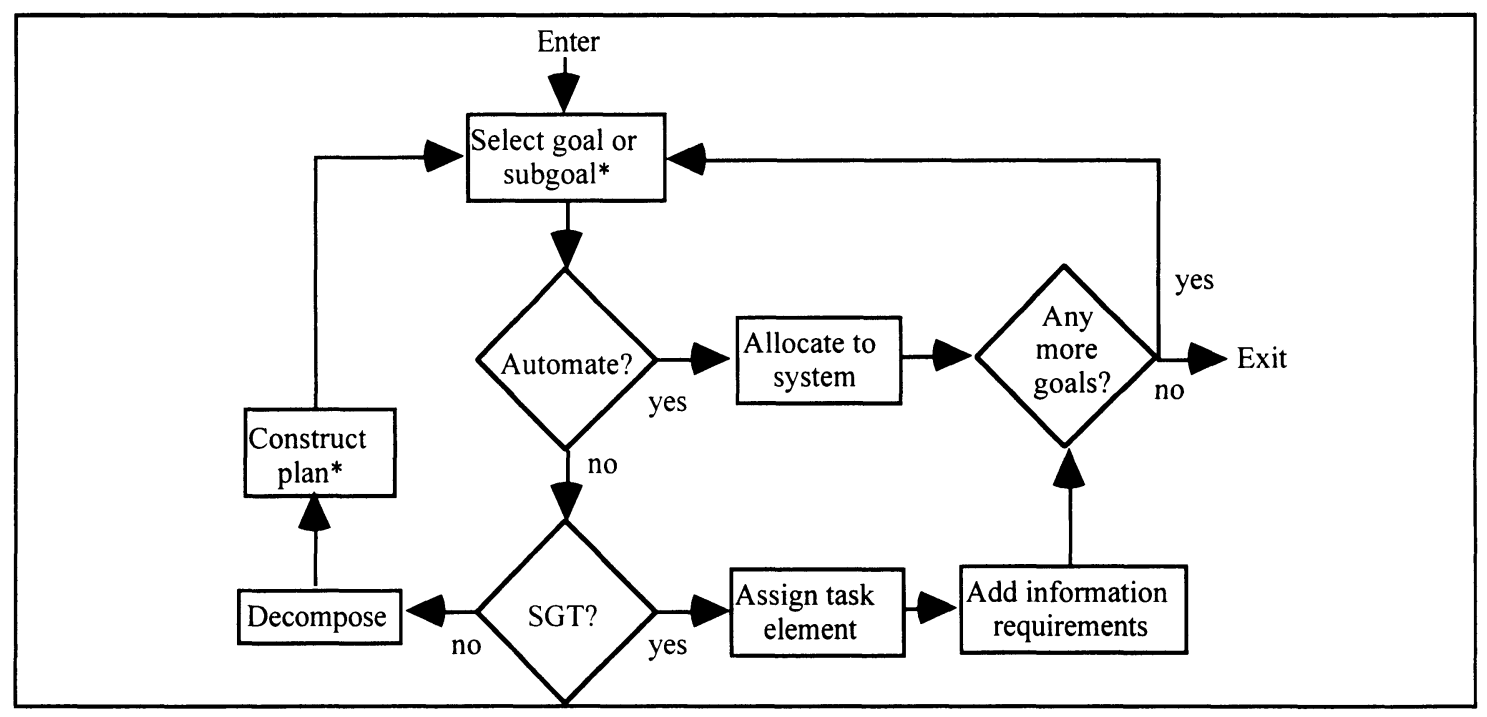

Figure 1. An illustration of the process of HTA translation and generation using the SGT scheme. Asterisks (*) indicate the points at which an existing HTA can be used to provide information. 
acceptable plan in terms of sequencing elements has been identified then it is recorded and the next goal, if any, should be selected. If the goal cannot be decomposed then attempts should be made to redescribe it or to loosen the goal constraints if possible.

Thus the stage labelled "Decompose" in Figure 1 covers a large number of different tasks involved in capturing the information required to produce an HTA. In particular, during this stage the analyst should collect information to determine the constraints acting on operational goals, the subgoals into which these goals can be decomposed and the possible sequences in which these subgoals can be carried out. If the analyst finds it impossible to decompose a goal then it might be necessary to consult the end-users to see if there is any way in which the constraints can be relaxed. For example, more funding may be made available.

If an HTA is available then the process is essentially the same except that the HTA can be used to provide input at certain stages. In particular, an HTA can be used to identify subgoals and their governing plan which can then be translated into sequencing elements. Therefore although the tasks of redescribing an existing HTA into SGT elements and constructing an HTA appear to be quite different, they are in fact essentially similar. The only difference is that if there is an existing HTA then it can be used to identify higher unexplored goals as well as subgoals and plans governing them.

\section{AN EMPIRICAL STUDY OF THE SGT NOTATION}

A preliminary evaluation of the SGT notation was carried out using 14 novice subjects. These subjects were given a pen and paper-based plan redescription task: they were presented with paragraphs containing descriptions of sequences of actions carried out on a fictional process control plant and were asked to express these sequences as plans using the sequencing elements of the SGT scheme. This task required subjects to work out the constituent tasks, their order and interdependencies and then write this using the SGT sequencing elements. Subjects were given sequences of varying levels of complexity: some were simple sequences of actions, others were more structurally complex sequences of interdependent actions.

The main result from this evaluation was that subjects' use of the SGT sequencing elements was by no means perfect. Given six plans to redescribe, on average subjects got only 3.3 correct on the first attempt, if they were allowed to attempt to correct their errors this figure rose to 4.5 correct. Over these six redescription tasks, subjects made, on average 4.6 errors, of which 2.25 were syntactic and 2.35 were semantic. Syntactic errors are defined as those in which an element is written down incorrectly or is used in an incorrect situation. Semantic errors are those in which the sequence is syntactically correct but is still wrong. The main types of syntactic errors made by subjects were to add extra items into the elements or to miss out parts especially when building complex embedded sequences of elements.

\section{A TOOL TO SUPPORT THE USE OF THE SGT SCHEME}

There are several reasons for constructing a tool to support the use of the SGT scheme. For example, Tse and Pong (1991) argue that a structured framework, such as that provided by a computer tool, to support requirements specification would allow the completeness and the consistency of the specification to be checked. In addition, Farooq and Dominick (1988) argue that design support tools can encourage different designers to use the same techniques, they can reduce time and cost and they can increase consistency between different interfaces.

Whilst these are all legitimate reasons for producing computer tools to support requirements specification we will focus here on two issues that are more specific to the SGT scheme: the problem of how to support use of the notation and the problem of how to support the methodology.

\subsection{Supporting the notation}

The notation of the SGT scheme requires subjects to use the sequencing elements to describe the interdependencies and ordering of tasks. The evaluation of the SGT notation reported above found that subjects tended to make several errors. In particular, they often missed out parts of the notation or added in extra items. Obviously, it is important that a computer tool should support the use of the SGT notation in such a way as to prevent these errors.

One possible solution is suggested by research carried out into the problem of supporting computer programming notations. Writing plans using the sequencing elements involves describing sequences of actions using a formal language. A similar task is involved in writing computer programs where the programmer has to translate sequences of activities into formal code. Sime, Arblaster and Green (1977a; 1977b) 
have investigated ways of reducing errors when subjects are asked to write programs using different forms of conditional statement. Sime et al. (1977a) investigated how subjects could be helped to make fewer syntactic errors when programming in a "mini-language" containing conditional statements. They found that subjects who were given a dictionary that automatically provided templates for whole conditional constructions solved more problems correctly at the first attempt than subjects who were taught a procedure for constructing programs. Sime et al. (1977b) obtained a similar result for subjects writing programs in another mini-language.

Sime et al. (1977a; 1977b) have demonstrated that an automated syntax can help computer programmers. It seems likely that this result can be generalised to the SGT scheme as there are parallels between the type of task used by Sime et al. and the task of using the sequencing elements. Both tasks involve using a formal, restricted language to describe relationships between different items. Thus an automatic syntax was incorporated into the SGT tool. When users of the tool select a sequencing element to put into a plan, they have to indicate the location of that element within the plan. An appropriate template is then inserted with spaces for filling in the subgoals. For example, if the user selects an S3 element to link two subgoals then the following template is written:

Then do together

-

And

-

The user must fill in two subgoals in the spaces given. In this way the user cannot either add extra items into the elements or miss out parts. In addition, the tool automatically checks that the points at which subgoals or elements are inserted are legitimate. For example, a subgoal can only be put into a plan to a location inside an element. Thus, the tool should support the notation and prevent user errors.

\subsection{Supporting the methodology}

The SGT tool must not only prevent errors in the use of the notation but it should also support the use of that notation within the methodology outlined above (Green, 1989). The SGT methodology can be split into two parts: checking to see if there are further unanalysed goals so that one can be selected and recording the information associated with the selected goal. In order to carry out the first of these processes, users of the tool will need a representation of the HTA which allows them to see which goals remain to be analysed in order to select one of them. In order to carry out the second process, users will need to be guided through the process of recording the appropriate information. The SGT tool was therefore designed with two main parts: (1) A Tree Diagram Window which contains a representation of the HTA which can be used to access information on individual goals and plans; (2) A Goal Information Window which guides the user through the process of inputting information related to a single goal.

The main component of the Tree Diagram Window is a representation of the HTA which is automatically updated as the HTA is built up. The representation consists of a hierarchical tree with each goal at each level represented by a node consisting of a box containing the goal's number and short label.

The Goal Information Window for an individual goal is opened when the user clicks on that goal in the Tree Diagram Window. This window guides the user through the process of recording the information associated with the current goal as defined by the methodology shown in Figure 1. Figure 2 gives a detailed illustration of exactly how this is achieved. For each goal the user is guided to record a short label, a longer description, an SGT element, if any, and the associated information requirements or, if no SGT element is assigned, the constraints, subgoals and plan. The parts of Figure 2 that are labelled with numbers map directly onto Figure 1 as follows:

(1) This is a dialogue window in which the user is asked if the goal can be automated. If they decide that it can be then this is recorded and no more information need be recorded for that goal. This supports the part of the process labelled "Automate?" in Figure 1.

(2) This indicates a window in which the user must chose the SGT element, if any, that describes the current goal. In this window the user is presented with a menu of the SGT elements. Further information on selected elements can be obtained as required. This supports the part of the process labelled "SGT?" in Figure 1.

(3) If an SGT element is chosen then it is assigned automatically to the goal. This supports the part of the process labelled "Assign task element" in Figure 1.

(4) Once an SGT element has been chosen then the user has to fill in answers to questions that elicit the information needs associated with the selected element. These information requirements are then filled into an appropriate template. Once the requirements have been recorded the information set for the goal is complete. This supports the part of the process labelled "Add information requirements" in Figure 1. 


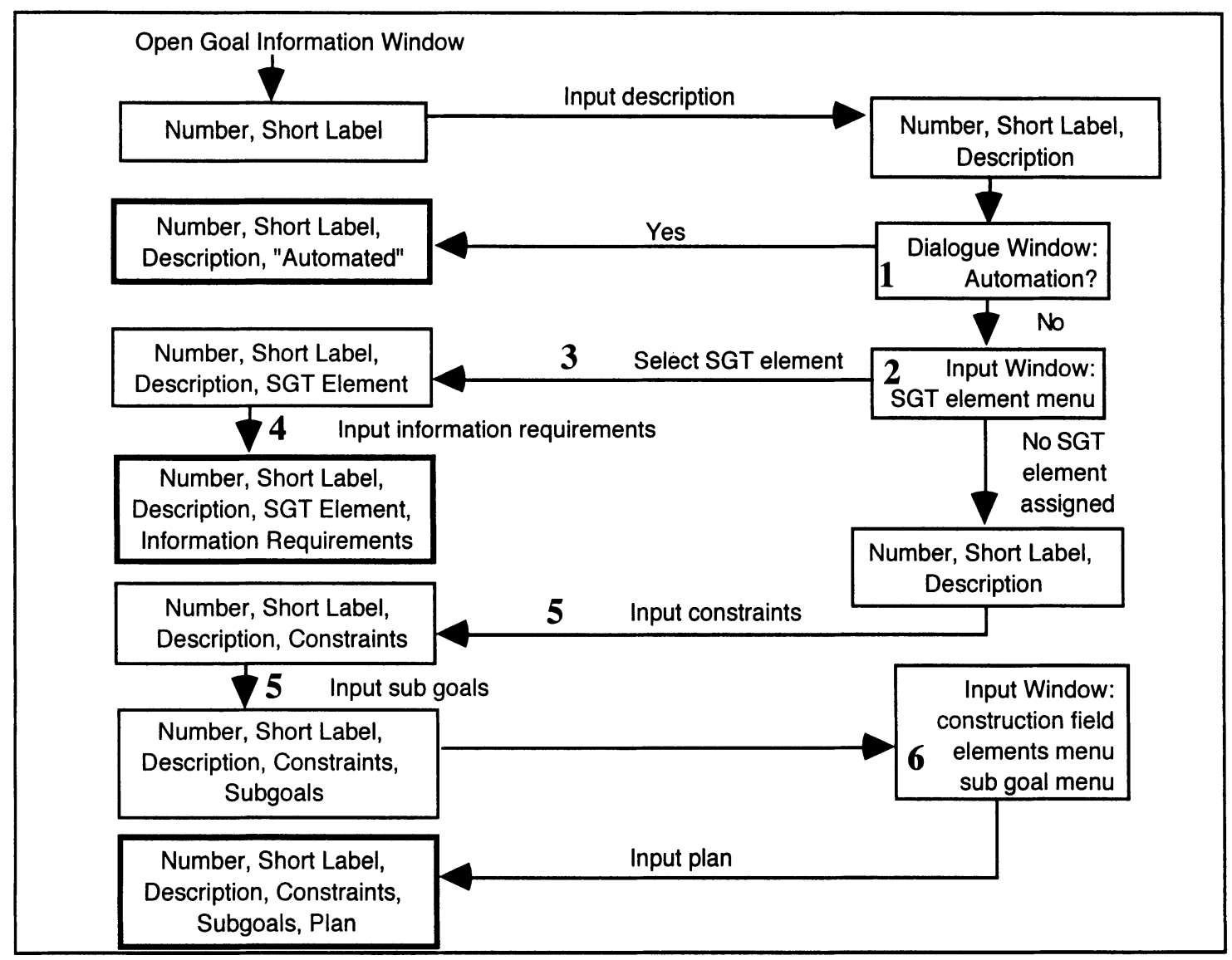

Figure 2. Illustration of the process of inputting individual goal information into the Goal Information Window.

(5) If no SGT element can be assigned to the goal then the user must specify the constraints acting on the goal. Once these have been specified the subgoals of the current goal must be identified. This supports the part of the process labelled "Decompose" in Figure 1. The numbering of subgoals is automated, so that the user is freed from having to work these out, and is able to concentrate on other parts of the analysis.

(6) Once the subgoals have been identified the user can open the Plan Construction Window. This contains a field into which the plan can be written, a menu of sequencing elements, information about the sequencing elements and a menu of the subgoals. The user constructs the plan by selecting a point in the construction field then selecting an element or a subgoal from one of the menus. The selected element or subgoal is then written to the chosen point as long as it is a legitimate location. This supports the part of the process labelled "Construct plan" in Figure 1.

The SGT tool thus not only guides the user through the process of inputting goal information but also provides the information that is needed for the various tasks involved in that process. In particular, information on the SGT and sequencing elements is provided at the points where the user has to select between these elements. The tool also guides the user through the process of collecting information by indicating at each point what type of information is required. 


\section{EVALUATION OF THE SGT TOOL}

There were two parts to this evaluation: the first part was designed to check whether the tool supported the use of the SGT notation whereas as the second part was carried out to check the extent to which the tool supported the SGT methodology.

\subsection{Evaluating support for the notation}

This evaluation was essentially a replication of the notation evaluation reported above. 16 novice subjects were used and they were given the same set of six plan redescription tasks. The only difference was that subjects were asked to carry out the task using the SGT tool rather than pen and paper.

Generally the results indicated that the SGT tool was successfully preventing errors in the use of the SGT notation. T-tests were carried out to test the significance of these effects. Out of the six tasks, subjects got, on average 4.5 correct on the first attempt. This is significantly more than the number of correct solutions achieved on the first attempt with pen and paper (3.33 correct, $p<0.05$ ). In addition, over the six tasks, on average subjects made only 1.83 errors, again this is significantly less than the number of errors made when using pen and paper ( 4.58 errors, $p<0.05$ ). Of these 1.83 errors, 0.33 were syntactic and 1.5 were semantic. The number of syntactic errors is significantly lower than the number made with pen and paper $(p<0.05)$ whilst there is no difference for the number of semantic errors. This shows that whilst the tool can help subjects to write down their interpretation of a sequence of actions with more accuracy it cannot increase the actual accuracy of the interpretation itself.

Thus, overall the features, such as templates, that were built into the SGT tool do seem to have had the desired effect of improving the level of accuracy with which the SGT notation is used to record sequences of activities.

\subsection{Evaluating support for the methodology}

This second evaluation was carried out to check whether the tool supports the SGT methodology as defined above. This evaluation was essentially a case study of an expert process control interface designer using the SGT tool. The designer was closely observed whilst he used the SGT tool to try and develop a user requirements specification for a computer tool to simulate and analyse a gas network.

In general the designer felt that the tool guided him through the use of the SGT scheme in a useful way. In particular, the tool made the subject think in detail about the sequences in which subgoals should be carried out and the conditions governing these sequences. This information is obviously central to good interface design. In addition, the user felt that it was easy to switch between different parts of the specification because of the good support provided by the tool: if he left a task half completed and then returned to it later he found it easy to pick it up again.

However, the subject did encounter a couple of difficulties in using the tool. Firstly, he felt that the way in which plans had to be constructed was too inflexible. The tool restricts plan construction to a strictly sequential process: the elements and subgoals have to be added into the plan in order. This aspect of the tool should therefore be altered to provide better support for the important task of specifying plans: users should be allowed to add elements and subgoals into the plan in any order.

The second problem that the subject encountered was that he felt that there was a lack of guidance on how to collect the information required by the SGT scheme. He thought that it would be useful if the tool could provide guidance on the type of information required, possible sources for that information and possible collection methods. As outlined above, the collection of information is an important aspect of the SGT methodology and the tool should be altered to provide better support for it.

Thus, in general the tool provided good support for the SGT methodology with just a couple of problems which were the inflexibility of plan construction and a lack of guidance on data collection. Both of these are important aspects of the SGT methodology (see Figure 1) and should be better supported by the tool. Carrying out a detailed evaluation in this way has highlighted these potential problems at an early stage.

\section{SUMMARY AND CONCLUSIONS}

The main conclusion to be drawn from this work is that care must be taken in designing usability-oriented tools. In particular, there should be two main inputs to the process of designing tools: (1) A high level analysis of the tasks involved in using the methodology should be carried out so that the tool can be designed to support them. In this way a tool can be produced that will guide users through the process of applying the methodology. (2) A lower level more detailed analysis of the use of the particular notation embodied within the methodology should also be carried out. The results 
from this can then be used to ensure that the tool prevents errors in using the notation as far as possible.

In addition to carefully designing the tool to support both the methodology and the notation, evaluations should be carried out at an early stage to check the extent to which the tool succeeds in these aims. In the case reported in this paper, it was found that the tool supported the use of the notation well, preventing errors found when subjects used pen and paper. The tool was also found to provide mainly good support for the methodology, although there were two important aspects of the methodology that were not well supported.

It is perhaps ironic that good design and careful evaluation of the usability of usability tools are often neglected. However, as this work demonstrates, both are of vital importance to the production of tools that will support designers and prevent simple errors. If the aim is to encourage the design of simple and effective system interfaces, then the tools that are used in interface development must be equally simple and effective.

\section{ACKNOWLEDGEMENTS}

The work reported in this paper was carried out as part of the first author's Ph.D. research and was funded by a studentship from British Gas plc. The second author's design research is currently supported by the ESRC Cognitive Engineering Initiative Grant No. L127251027.

\section{REFERENCES}

Annett, J., Duncan, K.D., Stammers, R.B. and Gray, M.J. (1971). Task Analysis. London: HMSO.

Ashworth, C. and Goodland, M. (1990). SSADM: a practical approach. London: McGraw-Hill Book Company.

Farooq, M.U. and Dominick, W.D. (1988). A survey of formal tools and models for developing user interfaces. International Journal of Man-Machine Studies, 29, 479-496.

Goel, V. and Pirolli, P. (1989). Motivating the notion of generic design within information-processing theory: the design problem space. AI Magazine, Spring, 18-36.

Green, T.R.G. (1989). Cognitive dimensions of notations. In A. Sutcliffe and L. Macaulay (Eds.), People and Computers V, (pp. 443-460). Cambridge: Cambridge University Press.

Guindon, R. (1992). Requirements and design of design vision, an object-oriented graphical interface to an intelligent software design assistant. In Proceedings of CHI'92, 499-506.

Harker, S.D.P., Olphert, C.W. and Eason, K.D. (1990). The development of tools to assist in organisational requirements definition for information technology systems. In D. Diaper (Ed.), HumanComputer Interaction - INTERACT '90. North-Holland: Elsevier Science Publishers B.V.

Jackson, M. (1980). The design and use of conventional programming languages. London: Academic Press.

Lansdale, M.W. and Ormerod, T.C. (1994). Understanding interfaces: a handbook of humancomputer interaction. London: Academic Press.

Olle, T.W., Hagelstein, J., Macdonald, I.G., Rolland, C., Sol, H.G., Van Assche, F.J.M. and Verrijn-Stuart, A.A. (1988). Information systems methodologies: a framework for understanding. Wokingham: AddisonWesley Publishing Company.

Parnas, D.L. and Clements, P.C. (1986). A rational design process: how and why to fake it. IEEE Transactions on Software Engineering, 12(2), 251-257.

Shepherd, A. (1993). An approach to information requirements specification for process control tasks. Ergonomics, 36(11), 1425-1439.

Shepherd, A. and Ormerod, T.C. (1992). Development of a formal method of user requirements specification for process plant displays. Report: Loughborough University of Technology, Loughborough.

Sime, M.E., Arblaster, A.T. and Green, T.R.G. (1977a). Reducing programming errors in nested conditionals by prescribing a writing procedure. International Journal of Man-Machine Studies, 9, 119126.

Sime, M.E., Arblaster, A.T. and Green, T.R.G. (1977b). Structuring the programmer's task. Journal of Occupational Psychology, 50, 205-216.

Simon, H.A. (1969). The sciences of the artificial. Cambridge, MA: MIT Press.

Tse, T.H. and Pong, L. (1991). An examination of requirements specification languages. The Computer Journal, 34(2), 143-152. 